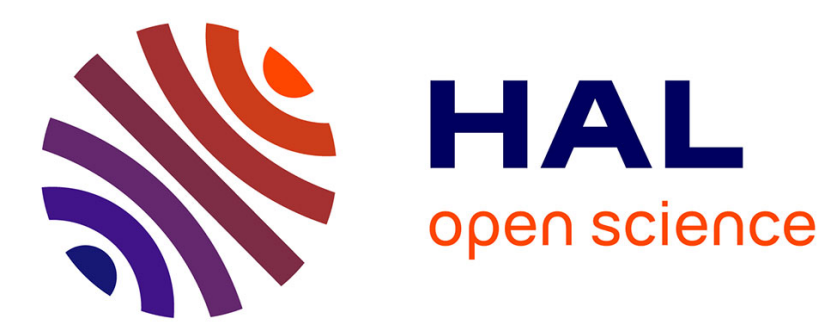

\title{
LA PROMULGATION DES LOIS PARLEMENTAIRES
}

Fabien Bottini

\section{To cite this version:}

Fabien Bottini. LA PROMULGATION DES LOIS PARLEMENTAIRES. Revue française de droit constitutionnel, 2008, 76, pp.761-784. 10.3917/rfdc.076.0761 . hal-02299555

\section{HAL Id: hal-02299555 \\ https://hal.science/hal-02299555}

Submitted on 27 Sep 2019

HAL is a multi-disciplinary open access archive for the deposit and dissemination of scientific research documents, whether they are published or not. The documents may come from teaching and research institutions in France or abroad, or from public or private research centers.
L'archive ouverte pluridisciplinaire HAL, est destinée au dépôt et à la diffusion de documents scientifiques de niveau recherche, publiés ou non, émanant des établissements d'enseignement et de recherche français ou étrangers, des laboratoires publics ou privés. 


\title{
LA PROMULGATION DES LOIS PARLEMENTAIRES
}

\author{
Fabien Bottini
}

Si l'attachement traditionnel de la France au culte de la « loi, expression de la volonté générale ${ }^{1}$ a longtemps justifié le refus d'un contrôle juridictionnel de constitutionnalité en droit interne, la création du Conseil constitutionnel sous la $\mathrm{V}^{\mathrm{e}}$ République a marqué une étape importante de la construction d'un État de droit au sein de la République 2 .

Mais qu'est-ce que l'État de droit ? Historiquement, une idée force - « une machinerie conceptuelle $»^{3}$ écrit Jacques Chevallier \héritée de la doctrine jusnaturaliste germanique. À l'analyse, un concept mou dont la signification varie selon la conception du droit à laquelle on se rallie. Alors que les positivistes juridiques en retiennent une définition formelle, en le présentant comme «un type d'organisation du pouvoir dans lequel existe une hiérarchie des normes $»^{4}$, les jusnaturalistes et les positivistes sociologiques ${ }^{5}$ doublent cette assertion d'une acception matérielle (ou substantielle). Selon eux, l'État de droit est celui dans lequel la règle de droit se trouve instrumentalisée afin de protéger les gouvernés contre l'arbitraire du pouvoir, en obligeant ses dépositaires à respecter les droits de l'homme (jusnaturalisme) ou les aspirations de la conscience collective (positivisme sociologique). Tandis que l'approche formelle des positivistes juridiques y voit une autolimitation de l'État par le droit sans réelle portée, l'approche matérielle des jusnaturalistes et des positivistes sociologiques en fait une « hétéro-limitation » contraignante pour les gouvernants. Or, ce caractère protecteur explique le succès de la notion outre Rhin en même temps que son internationalisation depuis la fin de la seconde guerre mondiale, la CJCE allant jusqu'à considérer la Communauté européenne comme une « communauté de droit » 6 .

\footnotetext{
${ }^{1}$ Rousseau (Jean-Jacques), Du Contrat social, rééd. Numilog, 2000, p. 69 ; DDHC, art. 6.

${ }^{2}$ C'est du moins ainsi que le président Giscard d'Estaing (« Allocution, prononcée au Conseil constitutionnel, le 8 novembre $1977 »$, http://discours-publics.vie-publique.fr) comprenait la transformation du juge de la rue Montpensier en gardien des libertés publiques (CC, Décision n ${ }^{\circ}$ 71-44 DC du 16 juillet 1971, Rec., p. 29, cons. $\left.\mathrm{n}^{\circ} 2\right)$.

${ }^{3}$ Chevallier (Jacques), L'État de droit, Paris, Montchrestien, 2003, p. 13.

${ }^{4}$ Troper (Michel), « Le concept d'État de droit », Droits, ${ }^{\circ} 15,1992$, p. 58.

${ }^{5}$ Cf. Lebreton (Gilles), Libertés publiques et droits de l'Homme, Paris, Armand Colin, 2005, p. 26.

${ }^{6}$ CJCE, 23 avril 1986, Les verts c/ Parlement européen, Rec., p. 1365, n 23.
} 
Si certains en ont déduit que « l'idée de réalisation de l'État de droit domin[ait] le droit constitutionnel moderne $\gg^{7}$, cette migration juridique ne s'est pas faite sans heurts. En écho au « coup de Charte » forgé par La Fayette, d'aucuns ont en effet dénoncé ce « coup d'État de droit $»^{8}$ en raison des errements dont s'est parfois accompagné son développement, tandis que, dans la lignée de Hans Kelsen ${ }^{9}$, d'autres continuent de lui dénier une quelconque vertu protectrice $^{10}$. Mais ces controverses ne font qu'illustrer la fertilité du débat doctrinal qui accompagne cette évolution.

Celle-ci paraît d'autant plus riche d'enseignements qu'elle oblige à envisager sous un jour nouveau des concepts pourtant anciens. La promulgation des lois est au nombre de ces règles qui, bien que séculaires, en viennent à devoir être revues à l'aune de son développement.

L'ancienneté de cette formalité est manifeste. Forgée sous l'Antiquité, à partir du latin «promulgare »- «publier»- la «promulgatio » désignait sous le Bas-Empire romain le commandement par lequel l'empereur obligeait ses agents à exécuter ou à veiller à l'exécution de ses édits ${ }^{11}$. De même, sous l'Ancien Régime, elle se concrétisait au travers de l'ordre donné par le Roi à ses «amé et féaux (...) tenant» ses «cours de Parlement »d' «observe[r] et de $\mathrm{f}\left[\right.$ aire] (...) observer $»^{12}$ ses ordonnances. C'est donc un mécanisme bien connu que la loi du 9 novembre $1789^{13}$ réintroduisait sous la Révolution, avant que les constituants successifs ne le fassent passer d'un régime à l'autre, que le texte considéré soit monarchique ${ }^{14}$ ou républicain ${ }^{15}$.

Sous l'effet du constitutionnalisme, la règle semble toutefois avoir changé de signification au tournant du XVIII ${ }^{\mathrm{e}}$ siècle, pour devenir un dénominateur commun à la plupart des démocraties constitutionnelles occidentales. Pour ne prendre que ces exemples, elle est

\footnotetext{
${ }^{7}$ Favoreu (Louis), «Le droit constitutionnel, droit de la Constitution et constitution du droit », RFDC, 1990, $\mathrm{n}^{\circ}$ 1, p. 79.

${ }^{8}$ Cayla (Olivier), « Le coup d’État de droit ? », Le débat, 1998, n 100, pp. 108-133.

${ }^{9}$ Kelsen (Hans), Théorie pure du droit, rééd. Paris, LGDJ, 1999, 367 p., p. 304.

${ }^{10}$ Troper (Michel), « Le concept..., op. cit., p. 63.

${ }^{11}$ Bonnet (Emile), De la promulgation (étude de droit public), Paris, A. Rousseau, 1908, pp. 17-18, n 5.

${ }^{12}$ Idem, pp. $18-19, \mathrm{n}^{\circ} 7$

${ }^{13}$ Décret du 9 novembre 1789, in Carette (Antoine-Auguste) (dir.), Lois annotées, t. 1, Paris, Adm. du Rec. gén. des 1. et arrêts, 1854 , p. 6.

${ }^{14}$ Const. du 3 septembre 1791, Chap. IV, Sect. 1ère, art. 1er ; Const. du 28 floréal an XII, art. 137 ; Charte du 4 juin 1814, art. 22 ; Charte du 14 août 1830, art. 18 ; Const. du 14 janvier 1852 (modifiée par le sénatus-consulte du 7 novembre 1852), art. 10 ; Const. du 21 mai 1870, art. 17.

${ }^{15}$ Const. du 5 fructidor an III, art. 128 s. ; Const. du 22 frimaire an VIII, art. 37 et 41 ; Const. du 14 janvier 1852, art. 10 ; Const. du 4 novembre 1848, art. 56 ; Const. Rivet du 31 août 1870, art. 2 ; L. const. du 25 février 1875, art. 3 ; L. const. du 16 juillet 1875, art. 7 ; Const. du 27 octobre 1946, art. 36 ; Const. du 4 octobre 1958, art. 10 .
} 
commune - outre au droit français - à la «due process of law» américaine ${ }^{16}$, au «Rechtsstaat» allemand ${ }^{17}$, au «stato di diritto » italien ${ }^{18}$ et à $l^{\prime}$ "Estado de derecho » espagnol $^{19}$. Or, sous leur empire, la promulgation ne sert plus seulement à «donne[r] l'ordre aux autorités publiques d'observer et de (...) faire observer ${ }^{20}$ l'acte voté par le Parlement, selon la formulation empruntée par le Conseil d'État à la terminologie de l'ancien droit ; elle vise avant tout à permettre au pouvoir exécutif, le plus souvent au chef de l'État, $\mathrm{d}^{\prime}$ « atteste[r] » de son «existence $»^{21}$, l'expression s'entendant d'une « déclaration officielle intervenant après l'élaboration d'une loi comme condition de son entrée en vigueur $»^{22}$.

Au sein de la République, cette récupération de la règle par l'État de droit n'est pas sans conséquences, puisqu'elle a récemment conduit les autorités normatives à lever deux des difficultés qui pesaient sur la notion depuis le $\mathrm{XIX}^{\mathrm{e}}$ siècle, en raison de la maladresse de rédaction de certains textes ${ }^{23}$.

La première découlait de l'article $1^{\text {er }}$ du Code civil dont il ressortait que «les lois [étaient] exécutoires dans tout le territoire français, en vertu (souligné par nous) de la promulgation qui en est faite ». L'expression «en vertu » était malheureuse, dans la mesure où elle tendait à faire croire que c'était la promulgation qui donnait sa force exécutoire à la loi, alors que cette force exécutoire découle de son adoption par le parlement ${ }^{24}$. Comme le relevait l'un des rédacteurs du Code civil en effet, «c'est après la promulgation (...), ce n'est pas en vertu de la promulgation, que la loi doit être exécutée ${ }^{25}$.

La seconde difficulté venait des dispositions d'une ordonnance du 27 novembre $1816^{26}$, reprises dans un décret du 5 novembre $1870^{27}$. Ces textes laissaient entendre que les

${ }^{16}$ Const. du 17 septembre 1787, préambule, art. 1er, Sect. 7, al. 2.

${ }^{17}$ L. fondamentale du 23 mai 1949, art. 82.

${ }^{18}$ Const. du ler janvier 1948, art. 73.

${ }^{19}$ Const. du 29 décembre 1978, art. 62 a.

${ }^{20}$ CE, Ass., 8 février 1974, Commune de Montory, Rec., p. 93.

${ }^{21}$ Idem.

${ }^{22}$ Guinchard (Serge) et Montagnier (Gabriel) (dir.), Termes juridiques, Paris, Dalloz, 2001, p. 718.

${ }^{23}$ Sur cette question, v. Carré de Malberg (Raymond), Contribution à la théorie générale de l'État, t. 1, rééd. Paris, éd. du CNRS, 1962, p. 429, n 143 s. Une troisième difficulté avait trait à la question de savoir si la promulgation donnait ou non sa date aux lois (Idem, p. 456-461, $\left.\mathrm{n}^{\circ} 152-153\right)$; question actuellement réglée dans le sens de l'affirmative par la juridiction administrative (CE, Ass., 8 février, 1974, Commune de Montory, Rec., p. 93).

${ }^{24}$ En ce sens, v. Bonnet (Emile), op. cit., p. 102, $\mathrm{n}^{\circ} 109$; Jèze (Gaston), «La promulgation des lois », RDP, 1918, p. 386 ; Carré de Malberg (Raymond), op. cit., p. 411, n 139 ; Sauvignon (Edouard), « La promulgation des lois : réflexions sur la jurisprudence Desreumeaux », RDP, 1981, 26 p., p. 999. Contra: Hauriou (Maurice), Principes de droit public, Paris, Sirey, 1910, XI-734 p., p. 448.

${ }^{25}$ M. le Tribun Andrieux, cité in Fenet (Pierre-Antoine), Recueil complet des travaux préparatoires du code civil, t. 6, Paris, éd. Au dépôt rue Saint-André-des-Arcs, 1827, p. 232.

${ }^{26}$ Ord. du 27 novembre 1816, in Carette (Antoine-Auguste), op. cit., t. 1, p. 969.

${ }^{27}$ Décret du 5 novembre 1870, Bull. des lois, XIIe série, 1870, 2e sem., n 29, p. 167, n 169 (abrogé par l'Ord. no 2004-164 du 20 février 2004, JORF, 21 février 2004, p. 3514, art. 6). 
expressions «promulgation » et «publication » étaient synonymes, puisqu'ils disposaient que «la promulgation des lois (...) résultera de leur insertion au $»^{28}$ bulletin ou au journal officiel. Cette assimilation s'expliquait à la fois par la finalité commune de ces termes - tous deux ayant étymologiquement pour objectif de porter quelque chose à la connaissance du public ${ }^{29}$ et par le fait qu'ils avaient souvent été confondus jusqu'à la Révolution ${ }^{30}$. Mais la loi et le décret précités des 9 novembre 1789 et 2-5 novembre $1790^{31}$ ainsi que nombre de Constitutions ultérieures les avaient clairement distingués, en faisant de la publication la conséquence de la promulgation $^{32}$. Aussi la majorité des auteurs s'accordait-elle pour leur donner une signification différente. Tandis que la promulgation tend à constater l'existence d'une loi faisaient-ils remarquer, la publication vise à la faire connaître à l'ensemble du corps social $^{33}$. En les assimilant, le législateur avait donc, «comme un écolier, confondu deux notions juridiques absolument distinctes $»^{34}$. Parant au plus pressé, la Cour de cassation avait estimé en 1874 que la «promulgation» visée au décret de 1870 dissimulait en fait une «publication $»^{35}$. Mais la clarification du droit positif rendait nécessaire une intervention du législateur.

Or, c'est au renforcement de l'État de droit que l'on doit d'avoir sorti les chambres du mutisme dans lequel elles s'étaient enfermées pendant plus d'un siècle. Même si elle ne s'en est pas expressément réclamée, cette évolution résulte bien de son développement, dès lors que c'est la nécessité de l' « accessibilité » et de $l^{\prime}$ « intelligibilité » ${ }^{36}$ de la règle de droit qui en découle qui a incité le gouvernement à « simplifier le droit ${ }^{37}$ de la promulgation par une ordonnance du 20 février $2004^{38}$.

Non content d'abroger le décret de 1870 , ce texte a réformé l'article $1^{\mathrm{er}}$ du Code civil afin de rendre plus lisibles les règles applicables en la matière. Cette évolution est manifeste à deux égards. D'abord, la nouvelle rédaction de cet article différencie nettement la

\footnotetext{
${ }^{28}$ Ord. du 27 novembre 1816, op. cit., p. 969, art. 1er ; décret du 5 novembre 1870, op. cit., p. 167, art. 1er.

${ }^{29}$ Tandis que le latin «promulgare » signifie «faire connaître en public », la «publicatio » désigne le fait de « rendre public » ou de «mettre à la disposition du public» (Dictionnaire étymologique de la langue latine, Paris, Klincksieck et Cie, 2001, resp. p. 538 et 542).

${ }^{30}$ Bonnet (Emile), op. cit., p. $19, \mathrm{n}^{\circ} 6$.

${ }^{31}$ Décret des 2-5 novembre 1790, op. cit., p. 76.

${ }^{32}$ Les Constitutions du 24 juin 1793 (art. 14) et de l'an III (art. 128 s.) semblent cependant avoir considéré le terme « promulgation » comme un synonyme de celui de « publication ».

${ }^{33}$ Esmein (Adhémar), Eléments de droit constitutionnel français et comparé, rééd. Paris, Panthéon Assas, 2001, p. 669 ; Barthélemy (Joseph) et Duez (Paul), Traité de droit constitutionnel, Paris, Dalloz, 1933, p. 740 ; Carré de Malberg (Raymond), op. cit., p. 439, n $^{\circ} 145$.

${ }^{34}$ Duguit (Léon), Traité de droit constitutionnel, t. 4, Paris, E. de Boccard, 2e éd., 1924, p. 627.

${ }^{35}$ Cass., 6 février 1874, D., 1874.1.187 ; ch. réunies, 22 juin 1874, D., 1874.1.323.

${ }^{36}$ CC, Décision n ${ }^{\circ}$ 99-421 DC du 16 décembre 1999, JORF, 22 décembre 1999, p. 19042.

${ }^{37}$ V. L. n ${ }^{\circ} 2003-591$ du 2 juillet 2003, JORF, 3 juillet 2003, p. 11193, art. 6.

${ }^{38}$ Ord. $\mathrm{n}^{\mathrm{o}} 2004-164$, op. cit., p. 3514, art. 1er et 6.
} 
promulgation de la publication, puisqu'il dispose désormais que les lois « entrent en vigueur dès leur publication » «si le décret de promulgation le prescrit $»^{39}$. Il met ainsi davantage en relief l'antériorité de cette formalité par rapport à l'autre, conformément à la jurisprudence constitutionnelle selon laquelle «la promulgation [de la loi] vaut ordre à toutes les autorités et à tous les services compétents de la publier sans délai ${ }^{40}$. Ensuite, le nouvel article $1^{\text {er }}$ lève les incertitudes qui pouvaient exister quant à l'origine de la force exécutoire des lois, puisqu'il cesse de dire qu'elles le deviennent «en vertu » de l'acte promulgatoire.

Ces efforts de clarification sont les bienvenus. Mais le droit positif semble animé d'un double mouvement en ce domaine, la portée de ces réformes se trouvant compromise par la multiplication des régimes promulgatoires.

Celle-ci fait suite à la volonté du pouvoir central de tenir compte des revendications autonomistes de certains territoires d'Outre-mer, le souci de la République de préserver l'intégrité de son sol l'ayant conduite à pousser très avant son processus de décentralisation en organisant deux nouveaux régimes de promulgations des lois. En allant du général au particulier, trois séries de règles se côtoient ainsi désormais.

La première, qui constitue le droit commun de la matière, renvoie à la promulgation des lois parlementaires ou référendaires ${ }^{41}$. La seconde, qui apparait comme une solution de compromis, a trait à la promulgation des «lois du pays » de la Nouvelle-Calédonie ${ }^{42}$. Quant à la troisième, qui constitue une solution véritablement dérogatoire, elle renvoie au régime de la promulgation des «lois du pays » de la Polynésie française ${ }^{43} 44$.

Au-delà de leurs ressemblances, ces régimes promulgatoires présentent en effet des divergences importantes. Ainsi, tandis que la promulgation des lois nationales et des « lois du pays » calédoniennes porte formellement sur des actes de nature législative ${ }^{45}$, celle des «lois

\footnotetext{
${ }^{39}$ Code civil, art. 1er (dans sa rédaction issue de 1'Ord. n ${ }^{\circ}$ 2004-164, op. cit., p. 3514, art. 1er).

${ }^{40}$ CC, Décision n ${ }^{\circ} 2001-448$ DC du 25 juillet 2001, JORF, 2 août 2001, p. 12493, cons. n 64 .

${ }^{41}$ Const. du 4 octobre 1958 , art. 10 et 11 .

${ }^{42}$ L. org. $\mathrm{n}^{\circ}$ 99-209 du 19 mars 1999, JORF, 21 mars 1999, p. 4208, art. 99 s.

${ }^{43}$ L. org. $n^{\circ}$ 2004-192 du 27 février 2004, JORF, 2 mars 2004, p. 4191, art. 64 s.

${ }^{44}$ Les deux dernières sortes de promulgation apparaissent comme un héritage de certaines ordonnances prises sous la Restauration, ces textes confiant aux gouverneurs le soin de «promulgue[r] les lois » (sur cette question, v. Lampué (Pierre), La Promulgation des lois et des décrets dans les territoires d'Outre-Mer, Paris, Guillemot et de Lamothe, 1956, 27 p.). Depuis la fin de la seconde guerre mondiale, les autorités normatives ont tendu à supprimer ces régimes dérogatoires en conséquence de leur volonté de simplifier le droit. V., par ex., décret $\mathrm{n}^{\circ}$ 47-1337 du 29 août 1947, JORF, p. 31 août 1947, p. 8664 ; L. n ${ }^{\circ}$ 55-1052 du 6 août 1955, art. 1-1 (dans sa rédaction issue de la L. $\mathrm{n}^{\circ}$ 2007-224 du 21 février 2007, JORF, 22 février 2007, texte $\mathrm{n}^{\circ} 2$, art. $144^{\circ}$ ). De sorte que la promulgation opérée par le chef de l'État s'impose désormais en principe à l'ensemble du territoire, y compris à l'Outre-mer (Cass., crim., 12 mai 1955, Rambeloson, Bull. crim., 1955, n 244, p. 442 ; TA, Nouméa, 28 juin 1995, Broisin, Juris-data, $\mathrm{n}^{\circ}$ 1995-051607).

${ }^{45} \mathrm{Du}$ moins, s'agissant des « lois du pays » calédoniennes, lorsqu'elles portent sur les matières législatives relevant de sa compétence (L. org. ${ }^{\circ}$ 99-209, op. cit., p. 4208, art. 107).
} 
du pays » polynésiennes est relative à des actes formellement administratifs ${ }^{46}$, même s'ils peuvent matériellement relever du domaine de la $\operatorname{loi}^{47}$. Alors que le terme prévu pour la promulgation des lois parlementaires et référendaires est un délai maximal ${ }^{48}$, celui qui conditionne la promulgation des «lois du pays » est aussi minimal : dans la mesure où cette formalité ne peut s'accomplir aussitôt après leur adoption ${ }^{49}$. Tandis que les lois nationales et les «lois du pays » calédoniennes ne s'imposent aux tiers qu'après que leur promulgation ait fait l'objet d'une publication ${ }^{50}$, les « lois du pays » polynésiennes leur sont opposables dès leur adoption ${ }^{51}$. Enfin, tandis que le décret de promulgation des lois parlementaires ne peut être contesté ${ }^{52}$, celui des «lois du pays » polynésiennes peut faire l'objet d'un «recours spécifique » devant le Conseil d'État ${ }^{53}$, la question restant en suspens pour les «lois du pays » calédoniennes, dans l'attente d'une éventuelle saisine des juridictions sur ce point.

En raison de la complexification du droit positif qu'elles entrânent, ces divergences soulèvent une question intéressante: n’est-il pas possible d'améliorer le régime de la promulgation des lois parlementaires ${ }^{54}$ à la lumière de celui des «lois du pays », de façon à renforcer les garanties offertes aux citoyens contre l'arbitraire éventuel du pouvoir exécutif ?

Cette question nous amène à nous interroger sur les implications de la notion d'État de droit. Or, aussi paradoxal que cela puisse paraître, celui-ci semble fonder la compétence de l'exécutif en la matière (I) en même temps qu'il justifie de la dépasser (II).

\footnotetext{
${ }^{46}$ CC, Décision n ${ }^{\circ} 2004-490$ DC du 12 février 2004, JORF, 2 mars 2004, p. 4225, cons. ${ }^{\circ} 75$.

${ }^{47}$ En ce sens, v. note Moyrand et Troianiello sous CE, Sect., 1er février 2006, Sandras et commune de Papara, RFDA, 2006, p. 282.

${ }^{48}$ Const. du 4 octobre 1958, art. 10, al. $1^{\mathrm{er}} ; 11$, al. 3.

${ }^{49}$ La promulgation des lois calédoniennes et polynésiennes doit intervenir :

- soit dans les dix jours de l'expiration du délai prévu pour contester leur validité devant l'instance juridictionnelle (ledit délai étant respectivement fixé à 10 jours après une nouvelle délibération en NouvelleCalédonie et à un mois après la délibération initiale en Polynésie française) ;

- soit dans les dix jours suivants la publication au Journal officiel calédonien ou polynésien de la décision l'autorisant (L. org. n 99-209, op. cit., p. 4208, art. 106 ; L. org. ${ }^{\circ}$ 2004-192, op. cit., p. 4207, art. 178).

${ }^{50}$ Vedel (Georges), Droit constitutionnel et institutions politiques, Paris, Les cours de droit, 1960, p. 1003 ; CE, 12 janvier 2005, Congrès de Nouvelle-Calédonie, AJDA, 2005, p. 555, concl. F. Donnat; note Moyrand et Troianiello sous CE, 15 mars 2006, Société Super Fare Nui, RFDA, 2006, p. 1121.

${ }^{51}$ CE, 15 mars 2006, op. cit., p. 1118 s. Comme le relèvent les commentateurs, cette surprenante solution est contestable et il convient d'être vigilant sur la suite que le CE entend donner à cette « jurisprudence ».

${ }^{52}$ CE, 8 février, 1974, op. cit., p. 93.

${ }^{53}$ CE, 22 mars 2006, Fritch et al., RFDA, 2006, p. 1111 s., note Moyrand et Troianiello.

${ }^{54}$ On laissera de côté dans les développements la question de la promulgation des lois constitutionnelles qui se pose en des termes différents, puisque, en la matière, le chef de l'État semble dans une position de « compétence liée » (Beaud (Olivier), La puissance de l'État, Paris, PUF, 1994, p. 255), en ce sens que « la promulgation a lieu de plein droit après son adoption définitive » (Idem, pp. 255-256).
} 
I. LA PROMULGATION, COMPOSANTE DE LA «CONCEPTION FRANÇAISE DE L'ÉTAT DE DROIT »

La promulgation semble rétrospectivement constituer un élément du Rechtsstaat, dans la mesure où elle permet de sanctionner les lois qui méconnaissent la hiérarchie des normes (A). Elle semble toutefois illustrer l'existence d'une vision française de l'État de droit, dès lors que, à travers la primauté de la norme suprême, c'est le respect de la souveraineté populaire qu'il s'agit d'assurer (B).

\section{A. UNE GARANTIE DE LA PREEMINENCE FORMELLE DE LA CONSTITUTION}

Depuis le XVIII $^{\mathrm{e}}$ siècle, la promulgation semble se justifier par le souci du constitutionnalisme d'assurer la prééminence formelle de la Constitution, de façon à éviter l'arbitraire, outre de l'Exécutif, du Législatif. Cette considération a pu être occultée par le principe de séparation des pouvoirs, sur lequel la promulgation s'est trouvée fondée au XIX ${ }^{\mathrm{e}}$ siècle $^{55}$. Mais, au travers de ce principe, c'est bel et bien la Constitution qu'il s'agissait de faire respecter puisque, historiquement, la séparation des pouvoirs n'a elle-même d'autres finalités que d'éviter le despotisme des gouvernants, en leur imposant le respect des principes fondamentaux inscrits dans le texte suprême.

Entre autres conséquences, le baron de la Brède en a déduit la nécessité d'investir le chef de l'Exécutif du pouvoir de «prendre part à la législation par sa faculté d'empêcher » ${ }^{56}$, sous-entendu l'entrée en vigueur des lois parlementaires. Or, bien que le Chapitre VI de L'Esprit des lois ne dise pas clairement quelle forme ce pouvoir de blocage devait revêtir ${ }^{57}$, les Révolutionnaires et leurs successeurs ont vu dans la combinaison du droit de veto et de la promulgation le moyen d'équilibrer les fonctions exécutive et législative.

\footnotetext{
${ }^{55}$ Ducrocq (Théophile), « De la formule actuelle de promulgation des lois », Revue générale du droit, 1877, p. 6 ; Cours de Droit administratif, t. 1, Paris, Ernest Thorin, 1881, p. 18, n 21 ; Esmein (Adhémar), op. cit., p. 669.

${ }^{56}$ Montesquieu (Charles de Secondat, baron de la Brède et de), De l'Esprit des lois, t. 1, Paris, Gallimard, 1995 , p. 338.

57 Montesquieu n'y emploie en effet ni le terme de veto, ni celui de sanction; il se réfère simplement à la « faculté d'empêcher » du chef de l'État (Idem).
} 
Ils ont tout d'abord conçu le droit de veto du chef de l'État comme la concrétisation de sa faculté d'empêcher l'entrée en vigueur des lois qui violent la Constitution au fond. Sous les régimes d'inspiration monarchique, ce moyen d'action était très efficace, dans la mesure où il se confondait avec le droit de sanction qui permettait au chef de l'État de s'opposer aux lois dont il contestait la constitutionnalité ${ }^{58}$. À l'image de Louis XVI - qui s'en était servi le 10 août 1792 pour s'opposer à deux décrets jugés indispensables à la sauvegarde des acquis révolutionnaires -, les monarques ont cependant été tentés d'en user pour imposer leurs vues aux citoyens. Aussi les Républiques ultérieures ont-elles tendu à limiter les prérogatives du chef de l'Exécutif en la matière. À compter de la II ${ }^{\mathrm{e}}$ République, la faculté reconnue au président d'opposer son veto aux lois matériellement inconstitutionnelles est ainsi devenue toute relative : puisque elle consiste désormais simplement à demander aux assemblées de réexaminer leur texte à l'aune de ses observations ${ }^{59}$. Depuis 1848 , le chef de l'État n'a ainsi plus qu'un «veto législatif temporaire $»^{60}$, beaucoup moins contraignant que le veto suspensif américain $^{61}$ qui avait inspiré le constituant de 1791. Que l’on considère les régimes monarchiques ou les régimes républicains toutefois, la possibilité du chef de l'Exécutif de désapprouver le contenu des lois parlementaires se trouve complétée par celle de veiller au respect de la procédure législative prévue par la Constitution.

En 1791, cette faculté se concrétisait également au travers de son droit de sanction ${ }^{62}$. Mais, à partir de 1795, c'est à son pouvoir de promulgation qu'il doit de pouvoir empêcher l'entrée en vigueur des lois qui méconnaissent la Constitution en la forme. Cette dimension de

\footnotetext{
${ }^{58}$ Const. du 3 septembre 1791, Titre III, Chap. III, Sect. 3 ; Charte du 4 juin 1814, art. 22 ; Charte du 14 août 1830, art. 18 ; Const. du 14 janvier 1852 (modifiée par le sénatus-consulte du 7 novembre 1852), art. 10 ; Const. du 21 mai 1870, art. 17. Si elle ne lui reconnaissait pas expressément de pouvoir de sanction, la Constitution impériale de 1804 en donnait implicitement un au chef de l'État, puisqu'il pouvait refuser de promulguer les lois sur proposition du sénat (Const. du 28 floréal an XII, art. 71 et 72).

${ }^{59}$ Const. du 4 novembre 1848, art. 58 ; Const. de Broglie du 13 mars 1873, art. 2, al. 2 ; L. const. du 16 juillet 1875, art. 7, al. 2 ; Const. du 27 octobre 1956, art. 36, al. 2 ; Const. du 4 octobre 1958, art. 10, al. 2.

${ }^{60}$ Prélot (Marcel), Institutions politiques et droit constitutionnel, Paris, Dalloz, 1969, p. 674.

Sous la Ve République, le chef de l'État a demandé à trois reprises une nouvelle délibération aux chambres : $1^{\circ}$ ) en 1983, afin de leur permettre de retirer la loi sur l'Exposition universelle de 1989 qui faisait candidater la France à l'organisation de cette manifestation (décret du 13 juillet 1983, JORF, 16 juillet 1983, p. 2206) ;

$2^{\circ}$ ) en 1985, afin de permettre au parlement de revoir la loi sur le statut de la Nouvelle-Calédonie, pour tenir compte de son invalidation partielle par le Conseil constitutionnel (décret du 9 août 1985, JORF, 10 août 1985 , p. 9149) ;

$3^{\circ}$ ) en 2003 , afin de soumettre l'article 4 de la loi relative à l'élection des conseillers régionaux et des représentants au Parlement européen ainsi qu'à l'aide publique aux partis politiques à une nouvelle délibération, également pour tenir compte de son invalidation par le Conseil constitutionnel (décret du 4 avril 2003, JORF, 5 avril 2003, p. 6070).

${ }^{61}$ Const. du 17 septembre 1787, Préambule, art. 1er, Sect. 7, al. 2.

${ }^{62}$ Const. du 3 septembre 1791, Titre III, Chap. III, Sect. 2, art. 10. Encore que cet article annonce la solution ultérieure en interdisant aux ministres de «sceller» ou de «promulguer » les lois sanctionnées au mépris des formes constitutionnelles.
} 
la formalité promulgatoire a été clairement mise en lumière par le Constituant de l'an III, puisque celui-ci a expressément subordonné son accomplissement au constat de «l'observation des formes prescrites » ${ }^{63}$ par la Constitution. Mais cet aspect de l'acte promulgatoire a par la suite été systématisé par les rédacteurs du Code civil. C'est ainsi que Portalis présentait cette formalité comme le moyen de faire «savoir que la loi existe et qu'elle existe comme loi ${ }^{64}$ tandis que ses collègues précisaient qu'elle avait pour «objet (...) de certifier $»^{65}$ la constitutionnalité de la loi, c'est-à-dire de vérifier qu'elle «est revêtu[e] de toutes les formes constitutionnelles $»^{66}$.

Ainsi, si elle se distingue du pouvoir de veto par son objet, en ce qu'elle ne permet pas au chef de l'État de contester la constitutionnalité matérielle des lois, la promulgation s'en rapproche par ses effets, dans la mesure où elle lui permet de s'opposer à l'entrée en vigueur de celles qui sont formellement inconstitutionnelles.

Cette dimension de la promulgation explique que la formule promulgatoire ait pu confier au président de la III $^{\mathrm{e}}$ République le soin de vérifier $1^{\circ}$ ) si la loi avait bien été délibérée et $2^{\circ}$ si elle avait été adoptée en termes identiques par les Chambres ${ }^{67}$. Même si, sous la V $\mathrm{V}^{\mathrm{e}}$, un décret modifié de 1959 a semblé limiter l'examen du chef de l'État au point de savoir si les assemblées ont bien «adopté ${ }^{68}$ la loi, la constitutionnalité de ce texte apparaît douteuse, dès lors que le Conseil constitutionnel a clairement réaffirmé depuis que la promulgation vise à établir si « la loi a été régulièrement délibérée et votée » ${ }^{69}$.

Ce qui précède montre que la promulgation vise à éviter l'arbitraire du législateur, dans la plus pure tradition du constitutionnalisme. À compter de la $\mathrm{III}^{\mathrm{e}}$ République, le mécanisme se justifie d'ailleurs par le rôle de gardien de la Constitution joué par le chef de l'État ${ }^{70}$. C'est manifeste sous la $\mathrm{V}^{\mathrm{e}}$, où elle constitue l'un des pendants de l'article 5 qui fait de lui le «gardien actif de la Constitution ${ }^{71}$, en lui confiant expressément le soin de « veille[r] » à son « respect ». Au-delà de l'acte par lequel le président « atteste l'existence de la loi $»^{72}$, la promulgation constitue ainsi une forme de contrôle de constitutionnalité. C'est

\footnotetext{
${ }^{63}$ Const. du 5 fructidor an III, art. 131.

${ }^{64}$ M. le conseiller d'État Portalis, cité in Fenet (Pierre-Antoine), op. cit., p. 350.

${ }^{65}$ M. le Tribun Andrieux, cité in Idem, p. 232.

${ }^{66}$ M. le Tribun Grenier, cité in Ibid., p. 377.

${ }^{67}$ Décret du 6 avril 1876, Bull. des lois, XIIe série, 1876, 2e sem., n 296, p. 226, n 5092.

${ }^{68}$ Décret $^{\circ}$ 59-635 du 19 mai 1959 (JORF, 20 mai 1959, p. 5107), modifié par le décret $\mathrm{n}^{\circ}$ 90-218 du 8 mars 1990 (JORF, 11 mars 1990, p. 3013), art. 1er, 2 et 3.

${ }^{69}$ CC, Décision n ${ }^{\circ} 85-197$ DC du 23 août 1985, JORF, 24 août 1985, p. 9816, cons. n 15.

${ }^{70}$ Quant à l'émergence de ce rôle de gardien, v. Conac (Gérard), « Article 5 », in Luchaire (François) et Conac (Gérard) (dir.), La Constitution de la République française, Paris, Economica, 1987, p. 239.

${ }^{71}$ Idem, p. 239.

${ }^{72}$ CE, 8 février, 1974, op. cit., p. 93.
} 
une sorte de «contrôle de constitutionnalité formel minimum » confié au chef de l'exécutif ${ }^{73}$, la loi ne pouvant exister qu'autant «qu'elle a été votée par le parlement dans les conditions requises par la Constitution $»^{74}$.

Pour cette raison, la promulgation semble constituer un élément du Rechtsstaat français. De nos jours il est vrai, un consensus existe pour dire que la réalisation d'un véritable État de droit passe par le recours au juge et l'avènement d'une justice constitutionnelle $^{75}$. Mais il ne faut pas oublier que, jusqu'au milieu du XIX ${ }^{\mathrm{e}}$ siècle, le Rechtsstaat a tendu à confier aux autorités politiques le soin de faire office de $«$ juge ${ }^{76}$ pour assurer le respect de la hiérarchie des normes. Ce n'est en effet qu'à partir de 1848 que la doctrine germanique a commencé à défendre l'idée d'une substitution de moyens proprement juridictionnels aux moyens politiques de règlement des conflits ${ }^{77}$. Or, si sous la République de Weimar cette idée s'est trouvée positivée ${ }^{78}$, nombre d'auteurs considéraient alors l'interdiction faite au chef de l'État de promulguer les lois élaborées en violation de la Constitution ${ }^{79}$ comme une garantie suffisante du respect de la hiérarchie des normes ${ }^{80}$.

À l'analyse toutefois, le contrôle promulgatoire semble traduire l'existence d'une vision française de l'État de droit, dans la mesure où, à travers la Constitution, c'est la souveraineté populaire qu'il s'agit de faire respecter.

\section{B. UN GAGE DU RESPECT DE LA SOUVERAINETE POPULAIRE}

\footnotetext{
${ }^{73}$ Voilà qui relativise l'idée selon laquelle le contrôle de constitutionnalité « représente (...) un développement profondément étranger à la tradition républicaine française » (Cohen-Tanugi (Laurent), La métamorphose de la démocratie française, Paris, Folio, 1993, p. 124). D'autant que, sous la Révolution, certains parlementaires avaient défendu l'idée d'un tel contrôle par le chef de l'État. C'est ainsi que, le 7 septembre 1789, le député Sillery avait invité le Roi à s'《 opposer avec courage contre toutes les infractions que l'on voudrait (...) faire »à la Constitution, même si elles étaient le fait des parlementaires (Arch. parl., t. 8, p. 600).

${ }^{74}$ Massot (Jean), « Article $10 »$, in Luchaire (François) et Conac (Gérard), op. cit., p. 402.

${ }^{75}$ Dans son discours précité de 1977, le président Valéry Giscard d'Estaing, fort de son rôle de gardien de la Constitution, avait ainsi défini « l’État de droit » comme «celui dans lequel chaque autorité, de la plus modeste à la plus haute, s'exerce sous le contrôle d'un juge, qui s'assure que cette autorité respecte l'ensemble des règles, de compétence et de fond, auxquelles elle est tenue ». Selon certains, cette définition lui aurait été soufflée par le doyen Vedel (Heuschling (Luc), op. cit., p. 383, $\mathrm{n}^{\circ} 391$ ).

${ }^{76}$ Georg Jellinek, cité in Idem, p. 94, n ${ }^{\circ} 88$.

${ }^{77}$ En ce sens, v. Ibid., pp. 73-95, $\mathrm{n}^{\circ}$ 67-88. En France, ce lien entre justice constitutionnelle et État de droit semble avoir été établi par Carré de Malberg (Ibid., p. 407, n 414).

${ }^{78}$ Tribunal de l'Empire (Reichsgericht), 5e ch. civ., 4 novembre 1925, RGZ, t. 111, p. 320.

${ }^{79}$ Const. de Weimar, art. 70.

${ }^{80}$ En ce sens, v. Heuschling (Luc), op. cit., p. 133, n ${ }^{\circ} 126$; pp. 330-331, n 326.
} 
Les deux éléments sur lesquels portent le contrôle opéré via la promulgation - la réalité de la délibération des assemblées et l'effectivité de la navette parlementairetraduisent l'existence d'une vision française de l'État de droit, dans la mesure où ils manifestent le souci d'assurer la prééminence de la volonté générale exprimée au travers de la Constitution.

Dans la France de l'après 1789, la délibération des chambres poursuit en effet une double finalité. Elle vise tout d'abord à permettre aux citoyens d'exercer leur contrôle sur l'activité du parlement. C'est pourquoi les Révolutionnaires de 1793 ont perçu le caractère public des débats législatifs comme « la sauvegarde la plus sûre du peuple » ${ }^{81}$ et que les parlementaires de la $\mathrm{IV}^{\mathrm{e}}$ République ont considéré que ce serait «une lourde perte de garantie ${ }^{82}$ si les particuliers perdaient le bénéfice de «l'intégralité d'une publicité démocratique à laquelle ils ont droit ${ }^{83}$. Car la délibération leur permet de connaître les motifs qui ont déterminé les chambres et d'apprécier la légitimité de leur action. Elle vise ensuite à dégager ce que commande l'intérêt général, en obligeant des parlementaires d'horizons politiques différents à échanger leurs points de vue sur les questions de société.

C'est encore ce dernier objectif que poursuit le bicamérisme, puisqu'il tend à assurer une meilleure représentativité du corps social, en faisant en sorte que chaque assemblée tempère les ardeurs de l'autre. C'est la raison pour laquelle il convient de s'assurer que la loi a bien été votée par le parlement : parce qu'il faut éviter qu'une des chambres ne légifère seule lorsque cette possibilité ne lui est pas constitutionnellement reconnue, sauf à faire de la loi la seule expression des intérêts particuliers de ses auteurs.

On comprend dès lors que la promulgation soit une garantie importante de la souveraineté des citoyens. Car, en garantissant la délibération des assemblées et le respect de la navette parlementaire, elle permet aux électeurs d'exercer leur contrôle démocratique sur l'activité du parlement en même temps qu'elle leur donne les moyens d'obliger ce dernier à rechercher ce que commande le bien commun.

Vu sous cet angle, le contrôle de constitutionnalité dissimulé dans l'acte promulgatoire semble donc opérer un lien entre État de droit et démocratie, le premier étant mis au service de la seconde. Cette union peut surprendre, tant elle peut sembler contre-nature. Selon la présentation traditionnelle en effet, l'État de droit se veut avant tout une doctrine libérale ${ }^{84}$ :

\footnotetext{
${ }^{81}$ Intervention de M. Hérault de Séchelles, Arch. parl., t. 67, p. 141.

${ }^{82}$ Idem.

${ }^{83}$ Intervention de M. Léo Hamon, JO Déb., CR Cons. de la Rép., séance du 17 mars 1954, p. 436.

${ }^{84}$ Chevallier (Jacques), op. cit., p. 53.
} 
c'est la traduction française de la théorie allemande du Rechtsstaat, dont l'objet est, non d'assurer le respect de la souveraineté populaire, mais de préserver la liberté de chacun, au besoin contre les excès du peuple ${ }^{85}$. Dans l'Allemagne d'aujourd'hui, cette solution s'explique par le souvenir des atrocités nazies. Celles-ci ayant en 1949 conduit le constituant d'outre Rhin à ériger l'État de droit en garantie ultime des droits de l'homme contre les «dérapages d'un parlement, voire d'un peuple entier ${ }^{86}$, Michel Fromont a justement pu « dire qu'avant d'être une démocratie (...), » l'Allemagne « est d'abord et avant tout un État de droit ${ }^{87}$. Il est à ce titre révélateur que la Loi fondamentale allemande institue un «ordre constitutionnel libéral ${ }^{88}$ et qu'elle ait été adoptée, non par le peuple lui-même, mais par ses représentants.

C'est pourquoi le paradoxe n'est qu'apparent. Car la réception de la théorie du Rechtsstaat dans l'ordre interne s'accompagne de son adaptation à la tradition juridique française $^{89}$. Or, au-delà de ses spécificités institutionnelles, celle-ci se caractérise sous la plupart des régimes qui se sont succédé depuis 1789 par la volonté de prendre davantage en compte les attentes des citoyens, «la souveraineté populaire» apparaissant comme «l'horizon constitutionnel indépassable $»^{90}$ de la France post-révolutionnaire. «Pense-t-on », écrivait à ce propos Alexis de Tocqueville, «qu'après avoir détruit la féodalité et vaincu les rois, la démocratie reculera devant les bourgeois et les riches ? S'arrêtera-t-elle maintenant qu'elle est devenue si forte et ses adversaires si faibles ? ${ }^{91}$. Non, à l'évidence. C'est pourquoi cette réception-adaptation semble catalyser le développement d'une conception alternative de l'État de droit, qui permette de le réconcilier avec la pensée démocratique.

Cette évolution se trouve facilitée par l'ambiguïté de la notion même d'État de droit. Aux termes de la définition la plus couramment admise, celui-ci a pour originalité de prôner

\footnotetext{
${ }^{85}$ C'est ce qui explique que le juge constitutionnel allemand estime le pouvoir constituant lié par « les principes juridiques fondamentaux qui sont antérieurs et supérieurs à tout droit écrit » (Décision Südweststaat (État du sud-ouest), BVerfGE, vol. 1, p. 61), dans la mesure où il «n'est pas, d'un point de vue théorique, immunisé contre le danger de vouloir enfreindre ces limites suprêmes de la justice » (Décision Handbuch des Verfassungsrechts (égalité des sexes), BVerGE, vol. 3, p. 229) (citées in Heuschling (Luc), op. cit., p. 159, n 153 , notes 332 et 333$)$.

${ }^{86}$ Idem, p. $143, \mathrm{n}^{\circ} 135$.

${ }^{87}$ Fromont (Michel), «Le juge constitutionnel (en RFA) », Pouvoirs, 1982, n 22, p. 42.

${ }^{88}$ L. fondamentale allemande du 23 mai 1949, art. 10, al. 2 ; 11, al. 2 ; 18; 21, al. 2 ; 73, al. 1 ; 87a, al. 4 ; 91, al. 1. Sans doute la terminologie exacte employée par ces articles est-elle celle d'un « ordre constitutionnel libéral et démocratique ». Mais la seconde place donnée au mot «démocratique » montre bien que la priorité du régime n'est pas d'assurer la souveraineté du peuple mais la protection de la liberté.

${ }^{89}$ En ce sens, v. Chevallier (Jacques), op. cit., p. 25.

${ }^{90}$ Jouanjan (Olivier), «La Constitution de 1791 dans la doctrine constitutionnelle libérale française du XIXe siècle », in Bart (Jean), op. cit., p. 440.

${ }^{91}$ Tocqueville (Alexis de), De la démocratie en Amérique, t. 1, rééd. Paris, Folio, 1998, p. 33.
} 
la soumission - effective - de l'État au droit ${ }^{92}$, en obligeant les dépositaires du pouvoir à répondre devant un juge de tout manquement à la hiérarchie des normes. Or, rien n'interdit de comprendre ce devoir de soumission comme l'obligation faite aux gouvernants de respecter la volonté générale telle qu'elle est exprimée au travers de la Constitution, lorsque cette dernière est l'œuvre du peuple souverain.

Sous la $V^{\mathrm{e}}$ République, cette différence essentielle avec la conception allemande s'explique dans la mesure où, contrairement à la Loi fondamentale d'outre Rhin, la Constitution n'a pas qu'une supériorité formelle. Si les autorités normatives doivent la respecter, c'est avant tout parce que, ayant été approuvée par le peuple à 79,25\% des suffrages exprimés $^{93}$, elle reflète les valeurs cardinales auxquelles les Français sont attachés ${ }^{94}$. Dès lors que la loi constitutionnelle a été adoptée par le peuple lui-même, il convient en effet de s'assurer que les lois parlementaires ne puissent la méconnaître. C'est pourquoi le Conseil constitutionnel est fondé à affirmer que «la loi »parlementaire «n'exprime la volonté générale que dans le respect de la Constitution » ${ }^{95}$. Et pourquoi la « conception française de la séparation des pouvoirs ${ }^{96}$ semble cacher en fait une «conception française de l'État de droit ${ }^{97}$ : parce que, contrairement au Rechtsstaat allemand qui n'a d'autre fonction que d'assurer le respect des droits et libertés de ses ressortissants, l'État de droit français vise prioritairement à assurer la primauté de la souveraineté du peuple, le respect de la liberté individuelle de chacun n'étant que la conséquence de sa volonté. On a ainsi l'impression qu'en droit français la pensée libérale se trouve comme instrumentalisée par la pensée démocratique.

Les implications de la promulgation montrent d'ailleurs qu'au-delà de la prééminence formelle de la Constitution, c'est la souveraineté populaire qu'il s'agit de faire prévaloir.

Car que se passerait-il si le chef de l'État décidait de promulguer un texte différent de celui qui a été adopté ou validé, ou refusait de promulguer une loi régulièrement votée par la représentation nationale? Sous la $\mathrm{V}^{\mathrm{e}}$ République, l'ancien président de la République,

\footnotetext{
${ }^{92}$ En ce sens, v. Gicquel (Jean) et Avril (Pierre), Droit constitutionnel, Paris, PUF, 1992, p. 49 ; Cohen-Tanugi (Laurent), op. cit., p. 147 ; Duhamel (Olivier), Le pouvoir politique en France, Paris, Seuil, 1993, p. 50 ; Chevallier (Jacques), op. cit., p. 16 etc.

${ }^{93}$ En métropole tout du moins (comm. X... sous « Constitution du 4 octobre 1958 », D., 1958, lég., p. 324).

${ }^{94}$ Celles-ci semblent d'ailleurs toujours d'actualité, puisque les citoyens ont porté au pouvoir en 2007 le seul candidat à l'élection présidentielle issu d'un parti de gouvernement qui ne s'était pas prononcé pour une nouvelle République.

${ }^{95}$ CC, Décision n ${ }^{\circ} 85-197$ DC, op. cit., p. 9817, cons. n 27.

${ }^{96} \mathrm{CC}$, Décision $\mathrm{n}^{\circ} 86-224$ DC du 23 janvier 1987, JORF, 25 janvier $1987, \mathrm{n}^{\circ} 21$, p. 925, cons. $\mathrm{n}^{\circ} 15 ; \mathrm{n}^{\circ} 2004-$ 492 du 2 mars 2004, JORF, 10 mars 2004, n 59, p. 4645, cons. n ${ }^{\circ} 98$.

${ }^{97}$ Chevallier (Jacques), op. cit., pp. 24-30.
} 
François Mitterrand a expressément dénoncé l'inconstitutionnalité de ce dernier cas de figure, le chef de l'État ne pouvant selon lui profiter de son pouvoir de promulgation pour s'opposer au fond au vote d'une loi et ainsi s'arroger un droit de veto qu'il n'a constitutionnellement pas : s'appuyant sur le rôle d'interprète de la Constitution qu'il tenait ès qualité de l'article 5 du texte de 1958, l'intéressé établissait en 1993 que «lorsque le parlement vote » régulièrement «une loi, » le président «devien[t] un notaire et quand, le délai qui [lui] est imparti » expire, il « doi[t] signer (...) sans quoi [il] serait en situation de forfaiture $»^{98}$. Or, son interprétation s'est par la suite implicitement trouvée validée par la décision de son successeur, le président Chirac, de promulguer la loi créant le «Contrat première embauche » $(\mathrm{CPE})^{99}$ tout en demandant aux autorités publiques de ne pas l'appliquer ${ }^{100}$. L'inconstitutionnalité de l'hypothèse d'un président promulguant délibérément un texte différent de celui qui a effectivement été voté ou validé est tout aussi manifeste. De prime abord, on pourrait croire que le danger d'un tel détournement se trouve relativisé par l'attitude des juridictions qui acceptent de rectifier les «erreurs matérielles » découlant de l'écart entre le texte adopté ou validé et le texte publié ${ }^{101}$. Mais, outre que la preuve d'une telle erreur n'est pas toujours facile à établir ${ }^{102}$, les tribunaux ne peuvent y remédier qu'autant qu'on le leur demande.

C'est pourquoi la pensée démocratique qui sous-tend l'État de droit français donne aux citoyens les moyens de lutter contre de telles violations de la procédure législative via leurs représentants au parlement. De façon préventive, ces derniers peuvent en effet menacer d'engager la responsabilité du chef de l'État sur le fondement de l'article 68. Sous les Républiques qui se sont succédé de 1848 jusqu'en 2007, les parlementaires auraient ainsi pu le menacer d'engager sa responsabilité politico-pénale en le mettant en accusation pour haute trahison $^{103}$. Depuis la réforme constitutionnelle du 23 février $2007^{104}$, ils peuvent toujours menacer de le destituer en engageant sa responsabilité politique. Et, si ces menaces ne suffisaient pas à obtenir sa coopération, ils pourraient toujours les mettre à exécution, afin de l'écarter du pouvoir ${ }^{105}$. À titre de comparaison, la marge de manœuvre du peuple allemand est

\footnotetext{
98 «L'entretien télévisé du président de la République », Le Monde, 16 juillet 1993.

${ }^{99}$ L. n ${ }^{\circ}$ 2006-396 du 31 mars 2006, JORF, 2 avril 2006, texte ${ }^{\circ} 1$, art. 8 (modifié par la L. n 2006-457 du 21 avril 2006, JORF, 22 avril 2006, texte $\mathrm{n}^{\circ} 1$, art. unique).

100 «Jacques Chirac promulgue la loi sur le CPE, mais repousse son application », Le Monde, 31 mars 2006.

101 CE, 9 mars 1936, Ministre des finances c/ société X..., Rec., p. 299 ; 21 décembre 1945, Ministre des finances c/ Sieur X..., Rec., p. 264. Sur cette question, v., Sauvignon (Edouard), op. cit., p. 1011.

${ }^{102}$ En ce sens, v. Laferrière (Julien), op. cit., pp. 147-150.

${ }^{103}$ Esmein (Adhémar), op. cit., p. 672 ; Bonnet (Emile), op. cit., pp. 78-79, n 83.

${ }^{104}$ L. const. $\mathrm{n}^{\circ} 2007-238$ du 23 février 2007, JORF, 24 février 2007, n 47, texte $\mathrm{n}^{\circ} 6$.

${ }^{105}$ L. fondamentale allemande du 23 mai 1949, art. 61, al. 2.
} 
plus réduite dans la mesure où, s'il peut faire pression sur ses représentants au parlement pour mettre le chef de l'État en accusation, ils ne peuvent l'écarter du pouvoir, dès lors qu'il revient à la Cour constitutionnelle de le juger.

Le cas de figure inverse - celui d'une majorité parlementaire profitant de sa position dominante pour inciter le chef de l'État à promulguer une loi formellement inconstitutionnelle - confirme d'ailleurs cette idée que l'État de droit français vise à assurer le respect de la souveraineté populaire. Car dans une telle situation, le chef de l'Exécutif serait constitutionnellement conduit à dissoudre la chambre basse. Or, là encore, c'est la volonté générale qui l'emporterait, puisque, autant la réélection des députés sortant vaudrait condamnation de son refus de promulguer la loi, autant leur mise à l'écart par les urnes traduirait le rejet de la loi inconstitutionnellement votée. En comparaison, le chef de l'État allemand n'est pas assuré de pouvoir en appeler au peuple, dès lors qu'il ne peut, en principe, exercer son droit de dissoudre la chambre populaire que sur proposition du chancelier ; que sa décision de la dissoudre doit être contresignée par ce dernier ou par les ministres compétents ; et que la chambre peut toujours «éteindre » son droit de dissolution, en élisant un autre chancelier fédéral à la majorité de ses membres ${ }^{106}$.

Ainsi perçu, le régime de la promulgation des lois met en lumière une autre de ces spécificités françaises : celle qui tend à voir en l'État de droit le moyen d'assurer le respect de la souveraineté du peuple. Paradoxalement toutefois, les régimes dérogatoires qui président à la promulgation des «lois du pays » montrent que la consolidation d'un «Rechtsstaat à la française » passe par un aménagement des règles traditionnelles.

II. LA « CONCEPTION FRANÇAISE DE L'ÉTAT DE DROIT », FACTEUR D'UNE REFORME DU REGIME DE LA PROMULGATION

Dans les faits, rien ne peut interdire au chef de l'État de promulguer une loi votée en violation de la norme suprême ou de se hâter de promulguer un texte voté par le parlement, de façon à d'empêcher l'opposition de saisir le juge constitutionnel.

\footnotetext{
${ }^{106}$ Ibid., art. 68 et 58 .
} 
Or, face à de telles lacunes, le développement de la vision française de l'État de droit semble tout à la fois justifier de remettre en cause l'immunité de juridiction de l'acte promulgatoire (A) et de renforcer les garanties contre l'arbitraire éventuel de l'autorité exécutive (B).

\section{A. LA REMISE EN CAUSE ANNONCEE DE L'IMMUNITE DE JURIDICTION DE L'ACTE PROMULGATOIRE}

Le développement de la vision française de l'État de droit semble annoncer un renforcement du contrôle de la validité de l'acte promulgatoire. Cette solution apparait en tout cas souhaitable en raison des graves inconvénients auxquels conduit le droit positif actuel.

Ceux-ci sont bien réels, dans la mesure où la promulgation donne au chef de l'État le moyen de cautionner l'arbitraire de la majorité parlementaire ou de promouvoir le sien propre, avec l'aide de cette dernière. Rien ne l'empêche en effet de promulguer une loi manifestement irrégulière car non délibérée ou votée dans les termes prévus par la Constitution ${ }^{107}$; tout comme rien ne l'empêche de promulguer un texte pourtant censuré par le juge constitutionnel, avec le soutien d'une majorité de députés ou sénateurs.

D'une part en effet, les seules sanctions à de tels détournements de la procédure ne peuvent être mises en œuvre qu'avec l'assentiment de la majorité parlementaire : puisque sans son accord, la responsabilité du chef de l'État ne peut être engagée ${ }^{108}$. D'autre part, l'intéressé ne devrait pas avoir de mal à obtenir le contreseing ministériel exigé par l'article 19 de la Constitution, dès lors qu'en de telles circonstances le gouvernement «marche » avec le chef de l'État et l'Assemblée nationale; et que les députés peuvent toujours contraindre les membres du gouvernement à coopérer : en menaçant d'engager leur responsabilité politique

\footnotetext{
107 Comme c'est arrivé sous la IIIe République. Cf. Laferrière (Julien), op. cit., p. 115, note 1 ; Sauvignon (Edouard), op. cit., p. 1006, notes 54 et 55.

${ }^{108}$ C'est là, selon nous, la grande faiblesse de la réforme constitutionnelle de 2007 : elle ne règle pas la véritable difficulté posée par le mécanisme antérieur, celle-ci tenant à la faculté donnée à une majorité parlementaire fidèle d'empêcher l'exercice des poursuites contre le chef de l'État, du moins le temps de son mandat. De ce point de vue, il aurait paru plus opportun d'ouvrir aux citoyens la possibilité d'autoriser eux-mêmes l'exercice des poursuites contre le président de la République ou de prendre l'initiative de sa destitution, en leur reconnaissant un droit de pétition doublé d'un droit de recall. Quoi de plus légitime en effet que de renforcer la souveraineté des citoyens pour remédier à la crise de la démocratie ? Sur cette question, v. Bottini (Fabien), $L a$ protection des décideurs publics face au droit pénal, Thèse, Le Havre, 2006 (à paraître à la LGDJ).
} 
collective sur le fondement de l'article 49 alinéa 2 de la Constitution; ou leur responsabilité pénale individuelle sur le fondement de ses articles 68-1 et suivants.

Sans doute la promulgation ne produira-t-elle pas les mêmes effets selon qu'elle portera sur un texte irrégulièrement adopté ou promulgué malgré la censure du juge constitutionnel. Car, dans ce dernier cas, l'article 62 de la Constitution interdit aux pouvoirs publics de «m[ettre] en application » les dispositions déclarées inconstitutionnelles. Mais, outre que l'interprétation restrictive dont cette disposition fait l'objet dans les faits relativise sa portée ${ }^{109}$, force est de constater qu'une telle prohibition n'existe pas lorsqu'on envisage le cas des lois promulguées bien qu'irrégulièrement votées. De sorte que de tels textes pourraient recevoir application en pratique.

C'est d'autant plus vrai que les juridictions ordinaires refusent - conformément à la solution retenue sous le directoire ${ }^{110}$ - d'apprécier la validité du décret de promulgation, motif pris de ce qu'il s'agit d'un acte de gouvernement ${ }^{111}$, ne pouvant «faire l'objet d'une action contentieuse $»^{112}$.

L'affirmation de la conception française de l'État de droit suppose donc de réformer le régime promulgatoire, afin d'assurer un plus grand respect de la souveraineté populaire. Dès lors qu'ils sont « tenus d'obéir à la loi », « les citoyens » devraient en effet avoir « le droit (...) d'en faire rétablir, s'il y a lieu, le texte authentique ${ }^{113}$.

Les juridictions pourraient ainsi s'estimer compétentes pour apprécier la validité de l'acte promulgatoire. À défaut, elles pourraient accepter de le contrôler chaque fois qu'il promulguerait un texte qui n'aurait pas été adopté par le parlement ou validé par le contrôle juridictionnel de constitutionnalité ${ }^{114}$.

\footnotetext{
${ }^{109}$ Cass., Ass. plén., 10 octobre 2001, Breisacher, Bull. crim., 2001, n 206, pp. 662-663 : la cour considère contre l'opinion d'Olivier Duhamel («Le point de vue du Conseil n'a pas d'effet en droit», Le Monde, 26 janvier 1999) -, que le considérant par lequel le juge constitutionnel a réglé la question du statut pénal des actes détachables de la fonction présidentielle (CC, Décision $\mathrm{n}^{\circ}$ 98-408 DC du 22 janvier 1999, JORF, 24 janvier $1999, n^{\circ} 20, p .1318$, cons. n 16 ) n'est pas un obiter dictum. Mais elle considère en même temps, conformément à l'analyse de Bruno Genevois, que ledit considérant ne s'impose aux juridictions ordinaires « qu'au regard des modalités d'introduction dans l'ordre interne de l'engagement international objet de la décision» («Observations complémentaires », RFDA, 1999, n 4, p. 720). Ce qui montre bien l'interprétation restrictive donnée à l'article $62 \mathrm{C}$. par les juridictions.

${ }_{110}$ Cass., 18 fructidor an V, cité par Laferrière (Julien), op. cit., p. 120 : «c'est au Directoire exécutif que la Constitution a délégué le pouvoir de faire promulguer les lois, et c'est sur lui seul, à cet égard, que repose la responsabilité (...). Les autorités subordonnées n'ont pas le droit de décider que l'acte qui leur est envoyé comme une loi par le Directoire n'a pas ce caractère ».

${ }^{111}$ CE, 3 novembre 1933, Desreumeaux, op. cit., p. 36, note Gros ; S., 1934, 3e partie, p. 9, note Alibert ; RDP, 1934, p. 649, note Jèze. Ce dernier avait d'ailleurs pressenti cette solution plusieurs années auparavant (Jèze (Gaston), « La promulgation..., op. cit., p. 386).

${ }^{112}$ CE, 23 septembre 1992, GISTI et MRAP, AJDA, 1992, p. 752.

${ }_{113}$ Note précitée Alibert sous CE, 3 novembre 1933, Desreumeaux, S., 1934, 3e partie, p. 9.

${ }^{114}$ En faveur d'une telle solution, v. Laferrière (Julien), op. cit., pp. 125-129.
} 
Le Conseil d'État a en tout cas ouvert la voie à une telle évolution, puisqu'il s'est reconnu compétent pour apprécier la validité des actes promulguant les «lois du pays » polynésiennes dans chacune de ces hypothèses ${ }^{115}$. De prime abord il est vrai, cette évolution s'est trouvée facilitée par le fait que l'acte promulgatoire ne pouvait en l'espèce être considéré comme un acte de gouvernement, puisqu'il ne concernait pas les relations Exécutif / Législatif $^{116}$, les autorités habilitées à voter et à promulguer ces « lois du pays » ayant toutes rang administratif ${ }^{117}$. Mais, dans la mesure où elle permet de faire prévaloir la volonté générale exprimée au travers de la Constitution sur les intérêts particuliers des détenteurs du pouvoir, la généralisation de cette solution aux actes irrégulièrement votés par le parlement paraît souhaitable. C'est la raison pour laquelle le développement de la conception française de l'État de droit devrait encourager les juridictions à mettre un terme à l'absence actuelle de recours contre l'acte promulgatoire.

Les intéressées pourraient ainsi revoir leur jurisprudence pour considérer la promulgation d'une loi irrégulièrement adoptée par les assemblées ou censurée par le contrôle de constitutionnalité comme un acte détachable. Un tel revirement serait conforme à la tendance actuelle du droit positif, consistant à mieux circonscrire les actes de gouvernement $^{118}$. Mais, plutôt qu'au Conseil d'État, cet examen pourrait être confié au Conseil constitutionnel. Le législateur-constituant pourrait ainsi étendre la jurisprudence par laquelle le juge constitutionnel s'est reconnu compétent pour contrôler certains actes de gouvernement ${ }^{119}$. Comme la haute juridiction a déjà accepté d'examiner la régularité formelle de la décision du chef de l'État d'user de la faculté que lui reconnait l'article 10 alinéa 2 de demander une nouvelle délibération au Parlement ${ }^{120}$, il suffirait d'étendre son contrôle au premier alinéa de cet article pour mettre un terme à l'injusticiabilité de l'acte promulgatoire.

Un tel changement constituerait un progrès. Mais il ne réglerait pas tous les problèmes que pose la promulgation. Une réforme d'une plus grande ampleur semble donc souhaitable pour prévenir l'éventuel arbitraire du président.

\footnotetext{
${ }_{115}^{115}$ CE, 24 octobre 2001, Gouvernement de la Polynésie française, Rec., p. 483 ; 22 mars 2006, op. cit., p. 1118.

${ }^{116}$ En ce sens, v. note précitée Moyrand et Troianiello, RFDA, 2006, p. 1114.

${ }^{117}$ Const. du 4 octobre 1958, art. 72 (issu de la L. const. $\mathrm{n}^{\circ}$ 2003-276 du 28 mars 2003, JORF, 29 mars 2003 , p. 5568, art. 5).

${ }^{118}$ CE, Sect., 25 septembre 1998, Mégret, AJDA, 1999, p. 240, note Lemaire ; Sect., 3 décembre 1999, Assoc. ornithologique de Saône-et-Loire, GAJA, ${ }^{\circ} 116$.

${ }^{119}$ CC, Décision n ${ }^{\circ}$ 85-197 DC, op. cit., p. 9816, cons. n 14 s. ; 11 juin 1986, Delmas, RDP, 1981, p. 1347, note Favoreu ; 25 juillet 2000, op. cit., p. 3. Dans ces affaires, le juge constitutionnel se reconnaît le droit de vérifier la régularité formelle des actes qui lui sont soumis.

${ }^{120}$ CC, Décision n ${ }^{\circ} 85-197$ DC, op. cit., p. 9816, cons. n 14 s.
} 


\section{B. L'ABANDON SOUHAITABLE DE LA FACULTE DU CHEF DE L'ÉTAT D'EMPECHER LA SAISINE DU JUGE CONSTITUTIONNEL VIA LA PROMULGATION}

Le développement de la vision française de l'État de droit passe également par la suppression de la possibilité dont le chef de l'État jouit en période de présidentialisme majoritaire d'empêcher via la promulgation l'opposition parlementaire ${ }^{121}$ de déférer une loi ordinaire au Conseil constitutionnel ${ }^{122}$. L'acte promulgatoire lui permet en effet de mettre l'intéressée dans l'impossibilité de saisir le juge constitutionnel, tant par voie d'action que d'exception.

La faculté du président d'empêcher l'opposition de saisir le Conseil par voie d'action résulte de la lecture combinée des articles 61 , alinéa 2 , et 10 , alinéa $1^{\text {er }}$, de la Constitution. D'après ce dernier, les autorités de saisine - notamment les 60 députés et/ou 60 sénateurs ayant bénéficié de la révision constitutionnelle de $1974^{123}$-, ne peuvent lui déférer une loi litigieuse qu'avant sa promulgation. Or, si l'article 10 donne un délai de 15 jours au chef de l'État pour l'accomplir, rien ne l'oblige à attendre cette échéance pour y procéder : puisqu'il s'agit d'un délai maximal et non minimal. Par conséquent, rien n’interdit au président de promulguer la loi à l'instant même où elle lui est communiquée, afin d'empêcher la saisine du juge constitutionnel, par la minorité parlementaire. La solution est certaine, puisque la suspension du délai de promulgation prévue par les textes ne court qu'à compter de la date à laquelle le Conseil se trouve saisi ${ }^{124}$. Et qu'elle a été clairement validée par le juge constitutionnel lui-même, ce dernier s'étant expressément déclaré incompétent pour se prononcer sur la constitutionnalité d'une loi qui lui était déférée postérieurement au décret de promulgation $^{125}$.

\footnotetext{
${ }^{121}$ Premier ministre, président de l'assemblée nationale, président du sénat et, depuis 1974, 60 députés et/ou sénateurs (Const. du 4 octobre 1958, art. 61, al. 2 issu de la L. const. du 29 octobre 1974, op. cit., p. 11035).

${ }^{122} \mathrm{La}$ question ne se pose pas pour les lois organiques, puisqu'en vertu des articles 46 al. 5 et 61 al. 1 er de la Constitution de 1958 celles-ci doivent automatiquement être soumises au juge constitutionnel.

${ }_{123}^{123}$ Const. du 4 octobre 1958, art. 61, al. 2 précité.

${ }^{124}$ Const. du 4 octobre 1958, art. 61, al. 5 ; Ord. modifiée n ${ }^{\circ}$ 58-1067 du 7 novembre 1958, JORF, 9 novembre 1958, p. 10131, art. 21.

${ }^{125}$ CC, Décision n ${ }^{\circ}$ 97-392 DC du 7 novembre 1997, JORF, 8 novembre 1997, p. 16255, cons. n 2. À l'appui de son dispositif, le Conseil relève que «la loi portant réforme du service national a été définitivement adoptée par le Parlement le 21 octobre 1997 ; qu'elle a été transmise au Gouvernement le même jour ; que le Président de la République a signé le 28 octobre 1997 l'acte portant promulgation de cette loi » et «que, par lettre du 29 octobre 1997, enregistrée le même jour au secrétariat général du Conseil constitutionnel, ce dernier a été saisi par plus de soixante sénateurs de ladite loi ».
} 
Le pouvoir de blocage du chef de l'État est d'autant plus redoutable qu'il lui permet également d'empêcher l'opposition de saisir le juge constitutionnel de la constitutionnalité d'une loi déjà promulguée par voie d'exception. En l'état actuel du droit positif, un tel contrôle est possible à l'occasion de l'adoption de la loi qui modifie, complète ou affecte le domaine d'un texte déjà en vigueur ${ }^{126}$. Mais, dès lors que cet examen ne peut être exercé qu'accessoirement à un recours par voie d'action, la promulgation donne au président le moyen de s'y opposer : puisqu'il lui suffit là encore de se hâter de promulguer le texte qui vient juste d'être adopté pour rendre impossible l'exercice de cette variante du contrôle de constitutionnalité.

Les verrous qui existent contre de tels détournements de la procédure apparaissent en tout cas insuffisants pour les prévenir en période de présidentialisme majoritaire.

Les deux premiers - la responsabilité du chef de l'État devant le parlement et la règle du contreseing ministériel - ne sont pas d'un grand secours, dès lors que la majorité de l'Assemblée nationale lui est acquise : puisqu'il y a peu de chance comme on l'a vu ${ }^{127}$ pour que cette dernière le destitue; et qu'elle peut contraindre les ministres à coopérer avec lui, en menaçant d'engager leur responsabilité politique ou pénale.

La dernière garantie, née de la pratique, apparaît tout aussi lacunaire. Elle consiste pour le secrétariat général du Conseil constitutionnel à informer son homologue du gouvernement $^{128}$ de l'intention de certains parlementaires de former un recours en inconstitutionnalité sur le fondement de l'article 61, afin qu'il suspende la procédure de promulgation $^{129}$. Mais elle n'est pas davantage satisfaisante, puisqu'elle n'est efficace que lorsque l'opposition parvient à recueillir les 60 signatures requises dans l'une ou l'autre des chambres dans les deux jours qui suivent le vote ${ }^{130}$. Un précédent montre en effet que, passé ce délai, rien ne garantit que l'information parviendra à temps au chef de l'État ${ }^{131}$. Surtout, ce rempart contre l'arbitraire de la majorité apparaît bien fragile, puisque tout repose en

\footnotetext{
${ }^{126}$ CC, Décision n ${ }^{\circ}$ 85-187 DC du 25 janvier 1985, JORF, 26 janvier 1985, p. 1138, cons. n 10.

${ }^{127}$ V. supra II,A.

${ }^{128}$ Lequel est chargé d'établir le texte dans les formes prescrites par le décret $\mathrm{n}^{\circ}$ 59-635 (op. cit., p. 5107), modifié par le décret ${ }^{\circ}$ 90-218 (op. cit., p. 3013); de le faire contresigner par les ministres responsables et le premier ministre ; et de le soumettre à la signature du président de la République (Guide pour l'élaboration des textes législatifs et réglementaires, Paris, La doc. fr., 2005, p. 123).

${ }^{129}$ Comm. X... sous CC, Décision n ${ }^{\circ}$ 97-392, Cah. CC, 1998, n 4, p. 17.

${ }^{130}$ Idem.

${ }^{131}$ CC, Décision n ${ }^{\circ}$ 97-392 DC, op. cit., pp. 16255-16256.
} 
définitive exclusivement sur le bon vouloir de l'Exécutif. Et que «tous ces contacts ont lieu sans formalisme particulier $»^{132}$.

La vision française de l'État de droit suppose de combler ces lacunes, afin de renforcer le respect de la volonté générale exprimée au travers de la Constitution. Si plusieurs réformes sont envisageables, il semble souhaitable de les cumuler, de façon à renforcer la souveraineté populaire.

$\left.1^{\circ}\right)$ La première, a minima, consiste à imposer au chef de l'État le respect d'un certain délai avant de promulguer la loi, ledit délai devant donner à l'opposition le temps de former son recours devant le juge compétent en dehors de toute situation d'urgence. Les «travaux préparatoires » de la Constitution de la $\mathrm{V}^{\mathrm{e}}$ République montrent que les constituants de 1958 n'étaient pas hostiles à un tel mécanisme, puisque le Comité consultatif constitutionnel (CCC) a accueilli favorablement l'amendement d'un de ses membres, M. Valentin, tendant à interdire à l'Exécutif de promulguer la loi dans les trois premiers jours de son adoption, de façon à éviter que l'« usage de son droit de promulgation » ne permette au chef de l'État de «dessaisir[] le Conseil constitutionnel ${ }^{133}$. Si le constituant a finalement décidé de laisser au législateur organique le soin de trancher la question et si ce dernier ne l'a pas fait en $1958^{134}$, celle-ci se pose à nouveau à partir du moment où il a consacré cette solution au tournant du $\mathrm{XXI}^{\mathrm{e}}$-siècle pour le régime promulgatoire des «lois du pays » calédoniennes et polynésiennes ${ }^{135}$. Alors, pourquoi ne pas la transposer aux lois de la République ?

$2^{\circ}$ ) La seconde réforme envisageable est plus ambitieuse. Elle consiste à compléter cette mesure par l'instauration d'un contrôle de constitutionnalité par voie d'exception, ouvert à chaque justiciable. En dépit de son caractère récurrent, une telle réforme ne semble toutefois pas pour demain. Car à l'hostilité traditionnelle des parlementaires envers elle s'ajoute désormais la réticence du président Sarkozy. «J'hésite » à opérer la réforme, a-t-il dit, «car l'avocat que je suis redoute une judiciarisation croissante de la société française ${ }^{136}$.

$\left.3^{\circ}\right) \mathrm{La}$ troisième alternative est plus révolutionnaire. Elle repose sur cette interrogation: faut-il véritablement maintenir le contrôle formel dissimulé dans l'acte promulgatoire entre les mains du chef de l'État? Dès lors en effet que la promulgation constitue une sorte de contrôle de constitutionnalité déguisé, pourquoi ne pas en confier

\footnotetext{
${ }^{132}$ Comm. X... sous CC, Décision n 97-392, op. cit., p. 17.

${ }^{133}$ Documents pour servir à l'histoire de l'élaboration de la Constitution du 4 octobre 1958, vol. II, Paris, La doc. fr., 1991, p. 183.

${ }^{134}$ Ord. $\mathrm{n}^{\circ}$ 58-1067 du 7 novembre 1958, op. cit., pp. 10129-10131.

${ }^{135}$ V. supra, note 49.

136 «Quel président je serai...», L'express, 27 mars 2007.
} 
l'exercice aux juges de la rue Montpensier? Outre qu'elle offrirait l'avantage d'unifier l'examen de la constitutionnalité des lois, cette solution déchargerait le chef de l'État d'une vérification qu'il n'opère de toute façon pas : «nul n'ignore » en effet qu'il « ne procède pas lui-même à la vérification des textes dont, par la promulgation, il atteste l'existence et la teneur; que cette vérification est l'œuvre des bureaux, » le président «se born[ant] à signer (...), est-il permis de dire sans irrévérence, (...) sans $1[\mathrm{a}]$ contrôler ${ }^{137}$. Surtout, une telle réforme aurait le mérite de prendre en compte l'évolution de notre société, dès lors que les raisons historiques qui ont traditionnellement justifié de confier cette formalité au chef de xécutif ne permettent plus de l'expliquer aujourd'hui.

Historiquement, la compétence du chef de l'État en la matière semble avoir constitué une autre conséquence du souvenir des abus des Parlements de l'Ancien Régime. Dans l'ancien droit, le «pouvoir judiciaire » avait profité de son pouvoir d'enregistrement pour s'arroger la faculté de confronter la loi monarchique avec la Loi fondamentale du Royaume. La défense de leurs privilèges ayant conduit ses membres à en profiter pour s'opposer aux réformes souhaitées par la monarchie pour adapter les institutions à la nouvelle réalité sociale, les Révolutionnaires n'ont eu de cesse de chercher à affaiblir les tribunaux ${ }^{138}$. La manifestation la plus célèbre de leur hostilité envers les juges reste l'interdiction qu'ils leur ont faite de «citer devant eux les administrateurs pour raison de leurs fonctions ${ }^{139}$. Mais celle-ci ne doit pas faire oublier la défense qui leur a parallèlement été faite de «prendre (...) part à l'exercice du pouvoir législatif » ou d' «empêcher ou » de «suspendre l'exécution des décrets du Corps législatif sanctionnés par le roi ${ }^{140}$. Car le transfert de la vérification de la constitutionnalité des lois des autorités juridictionnelles au pouvoir exécutif semble avoir été une conséquence de cette prohibition. C'est pourquoi on peut se demander s'il n'y a pas lieu de confier cette prérogative aux magistrats ; d'en déposséder le chef de l'État pour la donner aux juges $^{141}$ : car deux séries de mesures permettent désormais d'éviter que les intéressés n'en

\footnotetext{
${ }^{137}$ Laferrière (Julien), op. cit., p. 122.

${ }^{138}$ Sautel (Gérard) et Harouel (Jean-Louis), Histoire des institutions publiques depuis la Révolution française, Paris, Dalloz, 1997, p. 16, $\mathrm{n}^{\circ} 11$.

${ }^{139}$ Décret des 16-24 août 1789, Arch. parl., t. 8, p. 105, art. 13.

${ }^{140}$ Idem, art. 10. Cette interdiction s'est trouvée constitutionnalisée en 1791, par l'art. 3 du Chap. V de la Constitution du 3 septembre, qui rappelait que «les tribunaux ne peuvent (...) s'immiscer dans l'exercice du pouvoir législatif ou suspendre l'exécution des lois ».

${ }^{141}$ Cette proposition n'est pas sans rappeler les propositions qui avaient été faites à la fin du XIXe siècle en faveur de l'instauration d'un contrôle de constitutionnalité des lois, de telles propositions n'ayant « d'autre but que de rappeler nos juges à l'exercice d'un des devoirs normaux de leur magistrature que, sous l'influence de traditions surannées des temps révolutionnaires, ils [s'étaient] déshabitués de remplir » (Lambert (Edouard), Le
} 
profitent pour imposer leurs vues aux citoyens. La première tient au fait que les magistrats doivent répondre de leur judicature comme les autorités administratives de leur administration. Soumis à la volonté générale comme n’importe quel agent public, eux aussi s'exposent à des sanctions lorsque, outrepassant leurs prérogatives, ils commettent des fautes personnelles à l'occasion de l'accomplissement de leurs fonctions. Or, leur responsabilité civile et pénale peut être engagée dans les conditions du droit commun de ce chef, qu'ils appartiennent à l'ordre administratif ou judiciaire ou à la juridiction constitutionnelle ${ }^{142}$. La seconde garantie découle des prérogatives constituantes des pouvoirs législatif et exécutif, ces dernières leur permettant de se défendre contre les abus les plus graves de l'autorité juridictionnelle lato sensu, tandis que l'accroissement de la responsabilité pénale des parlementaires et des ministres devrait les inciter à n'en user que conformément à leurs aspirations.

Une telle évolution supposerait toutefois de modifier le contrôle de constitutionnalité en profondeur. En intégrant les autres propositions, on pourrait envisager la réforme suivante :

En amont de l'entrée en vigueur des lois, la promulgation pourrait être transférée au juge constitutionnel, de façon à renforcer son rôle de gardien de la Constitution. Il ne pourrait toutefois l'accomplir avant l'échéance du délai prévu pour permettre à l'opposition (ou aux autres autorités de saisine) de le saisir au fond de la constitutionnalité de la loi (ledit délai devant également permettre au chef de l'État de demander une nouvelle délibération aux chambres), deux hypothèses devant être distinguées à ce stade.

La première est celle où l'opposition (ou les autres autorités de saisine) ne saisit pas la juridiction constitutionnelle. Dans ce cas, son examen se limite au «contrôle de constitutionnalité formel minimum » que constitue la promulgation.

À l'inverse, la seconde est celle où la loi lui est déférée. Là, de deux choses l'une : ou bien le conseil déclare la loi constitutionnelle et il peut la promulguer; ou bien il estime que certains de ses articles méconnaissent la Constitution. Dans ce cas, il lui reviendrait de la promulguer chaque fois que les dispositions annulées n'en sont pas inséparables. Mais il ne pourrait le faire qu'à l'expiration d'un nouveau délai, ce dernier devant permettre au président

gouvernement des juges et la lutte contre la législation sociale aux États-Unis, rééd. Paris, Dalloz,

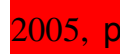

\section{5)}

${ }^{142}$ La seule exception à la règle tient à la responsabilité pénale des autorités qui participent à une procédure pénale, les poursuites ne pouvant être engagées à leur encontre que si l'illégalité de leur acte a d'abord été constatée par la juridiction répressive saisie de l'affaire dans le cadre de laquelle ils ont agi (C. pr. pén., art. 6-1). Sur cette question, v. Kerboal (Gwenola), La responsabilité des magistrats, Paris, PUF, 2006, 55 p. ; Bottini (Fabien), «La responsabilité personnelle des magistrats », RRJ, 2006, n 4, pp. 2193-2220. 
de l'une ou l'autre assemblée ou au chef de l'État de demander une nouvelle délibération du texte.

En aval de leur entrée en vigueur, les citoyens pourraient se voir reconnaître le droit de contester, par voie d'exception, la constitutionnalité des lois au fond, lorsque se poseraient des difficultés ayant échappé au contrôle a priori, que l'acte litigieux n'ait pas été soumis à l'examen du Conseil ou qu'il ne les ait pas perçues. Dès lors en effet que le contrôle a priori exercé par la juridiction constitutionnelle est un contrôle abstrait - c'est-à-dire que les juges sont saisis avant que ne se posent les difficultés concrètes d'application de la loi -, certaines d'entre elles peuvent lui échapper ${ }^{143}$.

L'efficacité d'une telle réforme reste cependant largement dépendante d'autres modifications, tenant à la fois à la composition et au mode de fonctionnement de la juridiction constitutionnelle ainsi qu'au statut de ses membres.

Pour lui donner son plein effet, il conviendrait tout d'abord d'augmenter le nombre de ces derniers ${ }^{144}$, de façon à permettre au Conseil de faire face à la surcharge de travail qu'entraînerait pour lui le transfert de la compétence promulgatoire et l'avènement d'un contrôle de constitutionnalité par voie d'exception centralisé. Une telle évolution semble cependant déjà inévitable, du fait de l'augmentation du nombre de lois qui lui sont déférées.

Il serait ensuite opportun de procéder à une plus grande judiciarisation de l'institution, par exemple en officialisant le caractère contradictoire des débats ou en organisant leur publicité, de façon à donner à son intervention toute la transparence qui lui est nécessaire ${ }^{145}$.

Enfin, il serait souhaitable de réformer le statut des juges constitutionnels. Outre qu'il conviendrait d'accroître les garanties de leur indépendance - notamment en subordonnant leur nomination à l'accord du parlement ou à des conditions de qualification dans le domaine juridique - , il paraîtrait judicieux de renforcer leur responsabilité disciplinaire. Les règles applicables en la matière n'ont en effet quasiment connu aucun remaniement depuis $1959^{146}$. Or, elles ne semblent plus être en rapport avec l'importance que l'institution a acquise au fil des ans, dès lors que les obligations qui leur incombent ont été sommairement définies par un décret pris en conseil des ministres et que ce texte confie aux juges constitutionnels le soin d'assurer leur propre discipline. Il paraîtrait ainsi opportun de préciser quelles obligations pèsent sur eux dans un décret en conseil d'État et d'externaliser leur contentieux disciplinaire.

\footnotetext{
${ }^{143}$ En ce sens, v. Hamon (Francis) et Troper (Michel), Droit constitutionnel, Paris, LGDJ, 2003, pp. 69-70.

${ }^{144}$ Cf. l'intervention de Pierre Mazeaud (JO Déb., CR AN, séance du 22 juin 1993, p. 2047).

${ }^{145}$ En ce sens, v. Fromont (Michel), «La justice constitutionnelle en France ou l'exception française », in Le nouveau constitutionnalisme, Mélanges en l'honneur de Gérard Conac, Paris, Economica, 2001, pp. 170-171.

${ }^{146}$ Décret modifié n ${ }^{\circ}$ 59-1292 du 13 novembre 1959, JORF, 15 novembre 1959, pp. 10818-10819.
} 
Pourquoi en effet ne pas le confier à un Conseil supérieur de la magistrature spécialement composé pour l'occasion, de façon à offrir toutes garanties d'indépendance, et pouvant se saisir, d'office ou à l'initiative des plus hautes autorités de l'État ou des citoyens, des fautes les plus graves dont ils peuvent se rendre coupables?

Faut-il aller aussi loin? Au point de vue théorique, la «conception française de la séparation des pouvoirs ${ }^{147}$ ne justifie-t-elle pas de maintenir la promulgation entre les mains du chef de l'État? Ces questions méritent réflexion. Car le transfert de cette prérogative au juge constitutionnel ne serait pas sans avantages. Outre qu'elle permettrait de revaloriser le rôle du parlement et de priver le président de la République du pouvoir de blocage dont il dispose aujourd'hui, une telle réforme semblerait plus conforme à la vision française de l'État de droit dans la mesure où, en rééquilibrant davantage les pouvoirs, elle permettrait d'assurer un meilleur respect de la souveraineté populaire. Elle pourrait même inspirer d'autres États et

Faut-il réformer la promulgation des lois ? Un constat contradictoire s'impose. D'un côté, les considérations théoriques qui justifient de recourir à l'acte promulgatoire n'ont rien perdu de leur pertinence, de sorte que la nécessité même de son existence ne saurait être contestée : dès lors qu'elle permet de s'assurer que la loi a bien été adoptée conformément à la Constitution, la promulgation constitue une garantie importante du respect de la souveraineté des citoyens par les autorités constituées qui ne saurait être remise en cause. Mais, d'un autre côté, il convient de tirer toutes les conséquences du développement de la vision française de l'État de droit, pour encadrer davantage l'exercice des pouvoirs présidentiels en la matière, voire, pourquoi pas, transférer l'exercice de cette formalité au Conseil constitutionnel. Dès lors en effet que l'hostilité traditionnelle de la classe politique à un contrôle juridictionnel de constitutionnalité des lois ne peut plus s'expliquer par son attachement à la souveraineté

\footnotetext{
${ }^{147}$ V. supra, note 133.
} 
parlementaire, le poids de la tradition semble devoir céder devant l'affirmation de la souveraineté populaire.

Une telle évolution suppose de réformer le Conseil constitutionnel. Mais, au-delà de son aménagement, la promulgation pose la question de la signification qu'il convient de donner à l'État de droit en France.

Ce dernier apparaît en cours de réalisation tant sa lente construction semble loin d'être achevée. Comme toute œuvre naissante, il se forme de façon empirique, par tâtonnements, sans vision d'ensemble. Il apparaît de ce fait en quête d'identité. Entre autres incertitudes, se pose celle de sa finalité. Car l'État de droit ne saurait se suffire à lui-même ; il n'est qu'un moyen au service d'une fin. Reste à déterminer ce qu'elle peut bien être dans le cadre de la République.

Plus influencés qu'ils ne le pensent probablement eux-mêmes par l'exemple allemand, les gouvernants français semblent tentés d'y voir le moyen d'assurer la défense des libertés individuelles. Cette conception de l'État de droit semble cependant affectée d'un vice fondamental qui la conduit en pratique à produire des effets inattendus au regard de la pensée libérale qui la sous-tend. Car loin de s'accompagner de l'État minimal nécessaire à son efficacité, le Rechtsstaat encourage l'intervention des autorités. De là la multiplication des normes protectrices, réglementant à tout va les différents domaines de la vie sociale, la judiciarisation de la société et la juridictionnalisation des relations humaines qui en découlent : car la protection des libertés individuelles passe par la multiplication des interdits, avec encore et toujours plus de normes. Il est vrai que cet État «n'a pas vocation à la simplicité ${ }^{148}$. Mais, combinée avec le développement du droit supranational, cette vision des choses met en péril la clarté du langage juridique et, avec elle, la défense des valeurs républicaines. Car le risque existe de voir le droit se transformer en une science ésotérique, hermétique aux profanes à qui il s'adresse pourtant. Et avec lui celui de l'avènement du «Government by the Judiciary» ${ }^{149}$, du «gouvernement des juges ${ }^{150}$, du «Richterstaat »(État des juges) également redouté de la doctrine allemande : le droit ne pouvant dans un tel système être que ce que les juges disent qu'il est, la souveraineté semble destinée à glisser inexorablement des citoyens aux élus et des élus aux magistrats si rien n'est fait pour en sortir. Le danger nous guette ainsi d'un État de droit contre-productif, constituant une menace pour la première des libertés démocratiques : celle, politique, qui permet aux

\footnotetext{
${ }^{148}$ Cohen-Tanugi (Laurent), op. cit., p. 203.

149 Boudin (Louis B.), « Government by Judiciary », Political Science Quaterly, 1911, n 26, p. 238.

${ }^{150}$ Lambert (Edouard), op. cit.
} 
citoyens de défendre leurs autres droits en participant directement à la Res publica, à la chose publique $^{151}$. Pour sortir de cette impasse, il convient d'assigner une autre finalité au Rechtsstaat français, afin d'éviter que la souveraineté populaire ne se trouve asphyxiée par le droit.

Le souvenir de l'impulsion égalisatrice donnée par les hommes de 1789 permet de déterminer ce que peut être l'alternative. Car, comme on l'a vu, ce qui compte avant tout dans notre pays ce n'est pas le respect des droits et libertés de chacun; c'est la défense de la souveraineté des citoyens, le respect de leurs droits et libertés étant la conséquence du règne de la volonté générale. Or, Vu sous cet angle, le «coup d’État de droit » ${ }^{152}$ appréhendé par certains n'en est pas vraiment un ; c'est avant tout un coup porté à l'État de droit ou plus précisément à la conception française de l'État de droit, dès lors qu'il conduit les juges à priver le peuple de son pouvoir de décision là où elle tend à le préserver.

Sans doute la consolidation de la vision française de l'État de droit français passe-telle par une plus grande responsabilité des magistrats vis-à-vis des citoyens: puisqu'il convient d'éviter que les juges ne profitent de leurs prérogatives pour étendre leurs compétences au détriment de celles du peuple. Mais dès lors qu'on parvient à trouver l'équilibre entre l'indépendance que requiert leur fonction et la possibilité de sanctionner leurs abus, l'État de droit devient un atout pour la démocratie.

Une troisième voie existe ainsi à l'alternative «gouvernement des élus ou gouvernement des juges »: celle démocratique, tendant à instrumentaliser l'autorité juridictionnelle afin de soumettre davantage les gouvernants au respect des attentes des citoyens.

Cette vision des choses permet de mieux cerner ce qu'est la version républicaine du Rechtsstaat : un instrument juridique destiné à préserver le caractère « démocratique ${ }^{153}$ des institutions, en assurant le respect de la «souveraineté nationale qui », en France, ne saurait qu' « appart[ir] au peuple ${ }^{154}$.

\footnotetext{
${ }^{151}$ La liberté-participation permet en effet au citoyen de s'approprier le pouvoir politique de sorte qu'il est « libre en ce sens qu'il n'obéit qu'à lui-même » (Lebreton (Gilles), Libertés publiques..., op. cit., p. 12).

152 Cayla (Olivier), op. cit.

${ }^{153}$ Const. du 4 octobre 1958, art. 1er.

${ }^{154}$ Idem, art. 3.
} 